\title{
Synergistic haemolysis test for presumptive identification and differentiation of Clostridium perfringens, $C$. bifermentans, $C$. sordellii, and C. paraperfringens
}

\author{
STEVAN M GUBASH
}

From the Department of Pathology, Division of Medical Microbiology, Kingston General Hospital, Kingston, Ontario, Canada K7L 2V7, and the Department of Microbiology and Immunology, Queen's University, Kingston, Ontario, Canada K7L 3N6

SUMMARY A new test for the presumptive identification of Clostridium perfringens, C. bifermentans, $C$. sordellii, and $C$. paraperfringens is described. The test is based on the synergistic haemolysis shown by the clostridia and group $\mathbf{B}$ streptococci on sheep and human and $\mathrm{CaCl}_{2}$-supplemented human blood agar. $C$. perfringens gave crescent-shaped synergistic lytic zones ( 7 to over $10 \mathrm{~mm}$ in length), and $C$. paraperfringens usually small-sized $(3 \mathrm{~mm})$, bullet-shaped reactions on all three types of media. C. bifermentans showed a horseshoe-shaped synergistic reaction only on human blood containing media, and $C$. sordellii only on $\mathrm{CaCl}_{2}$-supplemented human blood agar. $C$. perfringens type $A$ antiserum inhibited synergistic lytic activities of the four species. The test provided a reliable method for presumptive identification and differentiation of the four clostridial species and may obviate the need for the Nagler test.

Most clinical microbiology laboratories use the Nagler test ${ }^{123}$ for presumptive identification of Clostridium perfringens and other phospholipase $\mathrm{C}$ (lecithinase C) producing clostridia. Phospholipase C hydrolyses phosphatidylcholine (lecithin) to phosphorylcholine and a water-insoluble diglyceride 4 that manifests as a precipitation of a lecithovitellin complex' in the egg-yolk or human serum containing media. ${ }^{5}$ Phospholipase $\mathrm{C}$ activity of $C$. perfringens, $C$. bifermentans, $C$. sordellii, and $C$. paraperfringens can be inhibited by $C$. perfringens alpha-antitoxin. ${ }^{12} 6$ A further differentiation within this group of clostridia must be made by the use of several other criteria, such as lactose fermentation and production of gelatinase, tryptophanase, and urease. ${ }^{78} C$. absonum phospholipase $\mathrm{C}$ activity is only slightly inhibited by the alpha-antitoxin, ${ }^{6}$ and phospholipase C produced by some other clostridia, for example, $C$. novyi, is not antigenically related to that of $C$. perfringens. 49

The purpose of this study is to offer a simple test as an aid in the presumptive identification of $C$. perfringens and other clostridia that produce anti-

Received for publication 1 October 1979 genically related phospholipase $\mathrm{C}$. The test is based on the synergistic haemolysis due to the interaction of the CAMP-factor of group B streptococci and clostridial phospholipase $\mathrm{C}$ on sheep blood agar (SBA), human blood agar (HBA), and $\mathrm{CaCl}_{2-}$ supplemented human blood agar (CaHBA). The lytic phenomenon and its application in the presumptive grouping of beta-haemolytic streptococci were described earlier. ${ }^{10}$

\section{Material and methods}

\section{B ACTER I A}

In this study, 81 isolates of $C$. perfringens, 10 of $C$. bifermentans, 11 of $C$. sordellii, and four of $C$. paraperfringens were used. All isolates, except for five $C$. bifermentans and six $C$. sordellii, were obtained from routine clinical specimens from different patients in the Kingston General Hospital Microbiology Laboratory over a period of four years. D Muncey, of the Department of Microbiology and Immunology, Queen's University, Kingston, Ontario, donated one $C$. bifermentans (E41) and four $C$. sordellii (E16, E17, E18, and E19) strains; DE Mahony, of the Microbiology Department, Dal- 
housie University, Halifax, Nova Scotia, donated three C.bifermentans $(1124,2221 \mathrm{~A}$, and 2549) and one $C$. sordellii (9714) strain, and $M$ Magus, of the Special Reference Bacteriology Laboratory Services Branch, Toronto, Ontario, donated one $C$. bifermentans (SC286) and one C. sordellii (SL279) strain. The group B streptococcus was isolated from a routine clinical specimen.

Identification of the clostridia to the species level was based on Gram stain, spore formation, colony morphology, and type of haemolysis on human and sheep blood agar, half-antitoxin Nagler plate, lactose fermentation, gelatinase, tryptophanase, and urease activity. ${ }^{78}$ Motility tests were not performed on the majority of isolates. Routine clinical isolates were kept in chopped meat broth as stock cultures, and all donated strains came in lyophilised form. During the study all isolates were kept on blood agar plates in air at $4^{\circ} \mathrm{C}$ and subcultured on a fresh blood agar before testing.

\section{MEDIA}

Egg-yolk emulsion agar was a modified McClung and Toabe medium, ${ }^{3}$ using a fresh hen's egg and a tryptose blood agar base (Oxoid, London, UK).

For the synergistic haemolysis test, $15-20 \mathrm{ml}$ of the tryptose blood agar base containing $5 \%(\mathrm{v} / \mathrm{v})$ of citrated sheep blood (Woodlands Laboratory Ltd, Guelph, Ontario, Canada) or outdated whole human blood bank blood was poured into disposable Petri dishes $(15 \times 85 \mathrm{~mm})$. Washed erythrocytes were not used. Quality control of the plates was done as described earlier. ${ }^{10}$

To overcome chelating activity of sodium citrate in the whole human blood bank blood, and to supply necessary divalent cations for the phospholipase $\mathrm{C}$ activity and production ${ }^{411-13}$ additional sets of plates were supplemented by $\mathrm{CaCl}_{2} \cdot 2 \mathrm{H}_{2} \mathrm{O}$, $\mathrm{MgSO}_{4} \cdot 7 \mathrm{H}_{2} \mathrm{O}$, and $\mathrm{ZnSO}_{4} \cdot 7 \mathrm{H}_{2} \mathrm{O}$. Preliminary experiments indicated that useful concentrations varied in a relatively broad range for each tested salt, but final $\mathrm{w} / \mathrm{v}$ concentrations in the base medium of $0.066 \%$ for $\mathrm{CaCl}_{2}, 0.0066 \%$ for $\mathrm{MgSO}_{4}$, and $0.00066 \%$ for $\mathrm{ZnSO}_{4}$ were the most beneficial and were subsequently used in the study. Only one salt was added to one batch of the medium. When blood was mixed with the base medium containing additional $\mathrm{CaCl}_{2}$, defibrination was done by sterile glass beads to avoid formation of fibrin deposits in poured plates. After initial trials, $\mathrm{Ca}^{2+}$ supplemented $\mathrm{HBA}$ was chosen as a part of the set of media, and $\mathbf{M g}^{2+}$ supplemented HBA was used only when quality control of the new human blood batch showed some inferiority in the haemolytic patterns. In routine work, HBA could be easily supplemented if the well-dried plates were evenly flooded with $1 \mathrm{ml}$ of sterile aqueous solution of either $1 \% \mathrm{CaCl}_{2}$ or $0 \cdot 1 \% \mathrm{MgSO}_{4}$, allowed to soak for about 15 minutes at room temperature, and redried at $37^{\circ} \mathrm{C}$.

\section{TESTS}

In the half-antitoxin Nagler test, and the synergistic haemolysis test, $C$. welchii (perfringens) type $\mathrm{A}$ antiserum (Wellcome Reagents Ltd, Beckenham, UK) was used as a source of the alpha-antitoxin.

Half-antitoxin blood plates (SBA, HBA, CaHBA) were prepared by smearing a swab saturated with the C. perfringens alpha-antitoxin, undiluted or diluted $1: 6$ or $1: 10$ in sterile broth, over one-half of the surface of the dried plate, which was then redried at $37^{\circ} \mathrm{C}$. Two line inocula of group B streptococcus were made, one on the side without, and the other on the side with, the antiserum. Clostridial isolates were streaked at right-angles to the streptococcal lines, without touching, beginning on the antiserum-free side. Inoculated plates were incubated anaerobically overnight at $37^{\circ} \mathrm{C}$ in Gas Pak jars. Synergistic haemolysis reactions were read as 'large' (over 10 $\mathrm{mm}$ in length), 'intermediate' (5-10 mm), 'small' (less than $5 \mathrm{~mm}$ ), or 'negative' (if no synergistic haemolysis could be detected).

\section{Results}

The results of the synergistic haemolysis test and the Nagler test are given in the Table.

In all 81 isolates of $C$. perfringens, a positive synergistic haemolysis reaction was crescent-shaped on SBA, HBA, and CaHBA (Figs 1, 2, and 3). The lytic zone was consistently large in size $(15-18 \mathrm{~mm})$ in the 57 isolates on SBA, and in 63 on HBA or CaHBA. On HBA, the lytic zone was least intensive. Twentyfour isolates on SBA and 18 on HBA or CaHBA showed intermediate or large haemolytic zones ( 7 $\mathrm{mm}$ or more) at a different time or on different batches of agar.

Small-sized, bullet-shaped lytic zones ( $3 \mathrm{~mm}$ ) were given by three, and intermediate-sized, lytic zones ( 7 $\mathrm{mm}$ ) by one isolate of $C$. paraperfringens on all three types of blood agar.

None of the $10 C$. bifermentans or $11 C$. sordellii isolates showed any lytic synergism with group B streptococci on SBA (Fig. 1). However, on HBA (Fig. 2), a narrow, horseshoe-shaped clearing appeared inside the marginal zone of the darkened erythrocytes surrounding the usual haemolytic pattern of $C$. bifermentans and was similar to, but somewhat separated from, the latter haemolysis. Two $C$. bifermentans isolates were strongly haemolytic on HBA, but the overlapping field of the CAMPfactor:C. bifermentans haemolysis was helmetshaped, indicating the presence of the syngergism. 
Results of the Nagler test and synergistic haemolysis test with group B streptococci for presumptive identification and differentiation of $\mathrm{C}$. perfringens, $\mathrm{C}$. bifermentans, $\mathrm{C}$. sordellii, and $\mathrm{C}$. paraperfringens

\begin{tabular}{|c|c|c|c|c|c|}
\hline \multirow[t]{2}{*}{ Clostridial species } & \multirow[t]{2}{*}{ No. tested } & \multirow[t]{2}{*}{ Nagler test positive } & \multicolumn{3}{|c|}{ Synergistic haemolysis test positive on: } \\
\hline & & & $S B A$ & $H B A$ & $\mathrm{CaHBA}$ \\
\hline $\begin{array}{l}\text { C. perfringens } \\
C . \text { bifermentans } \\
C . \text { sordellii } \\
C . \text { paraperfringens }\end{array}$ & $\begin{array}{r}81 \\
10 \\
11 \\
4\end{array}$ & $\begin{array}{r}81 \\
10 \\
11 \\
4\end{array}$ & $\begin{array}{r}81 \\
0 \\
0 \\
4\end{array}$ & $\begin{array}{r}81 \\
10 \\
0 \\
4\end{array}$ & $\begin{array}{r}81 \\
10 \\
11 \\
4\end{array}$ \\
\hline
\end{tabular}

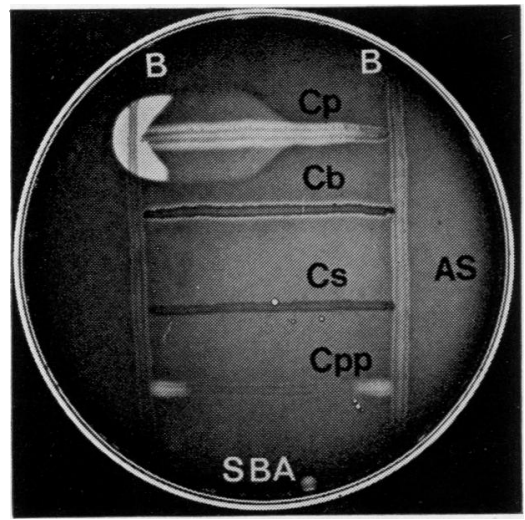

Fig. $1 S B A$. The right side was smeared with $\mathrm{C}$. welchii type A antiserum diluted 1:6 (AS). Group B streptococci inoculation lines $(B)$-left and right. C. perfringens $(C p), \mathrm{C}$. bifermentans, $(C b)$, C. sordellii (Cs), C. paraperfringens (Cpp). Note the crescent-shaped synergistic lytic zone, and the target haemolysis of $C p$ at the left, and complete inhibition of both at the right, no synergism by $\mathrm{Cb}$ and $\mathrm{Cs}$, and $\mathrm{a}$ small synergistic lytic zone of Cpp (left), only slightly inhibited by $A S$ (right).

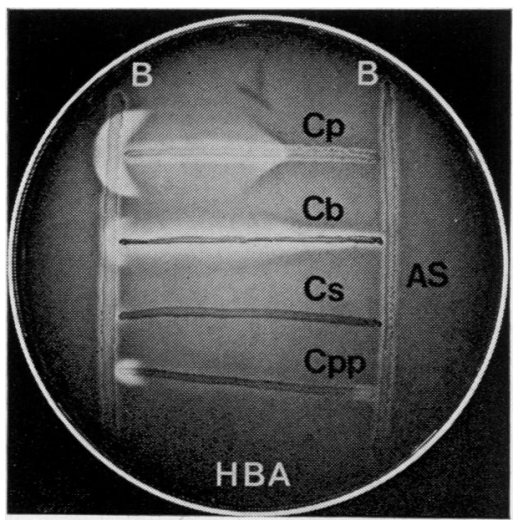

Fig. $2 H B A$. Inoculations, antiserum, and legend as for Fig. 1. Note appearance of horseshoe-shaped zone of clearing of $\mathrm{Cb}$ (completely inhibited at the right), and a more pronounced usual haemolysis than on $S B A$ (partially inhibited by $A S$ ).

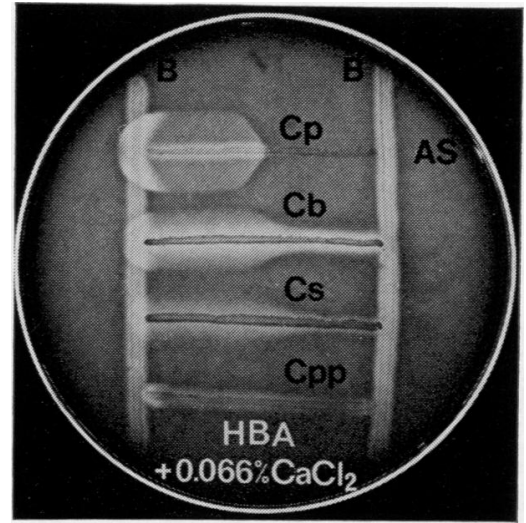

Fig. $3 \mathrm{HBA}$ supplemented with $0.066 \%$ of $\mathrm{CaCl}_{2}$. Inoculations, antiserum, and legend as for Fig. 1. Plates for Figs 1, 2, and 3 were inoculated with the same strain of either species (Cp-1, Cb-76, Cs-SL279, Cpp-8422). Note appearance of wide haemolysis and a weak synergistic clearing of $\mathrm{Cs}$ at the left. Inhibition by As is similar to that of $C b$.

C. sordellii isolates did not show any synergistic lysis on HBA (Fig. 2), but on CaHBA all 11 isolates gave similar, but less intensive, haemolytic and synergistic haemolytic patterns to those of $C$. bifermentans (Fig. 3). $\mathrm{ZnSO}_{4}$ or $\mathrm{MgSO}_{4}$ did not induce synergistic haemolytic activity of $C$. sordellii but did improve the target haemolysis of $C$. perfringens, and the usual and synergistic haemolysis of $C$. bifermentans was most enhanced by $\mathrm{CaCl}_{2}$ and $\mathrm{MgSO}_{4}$. None of the haemolytic reactions was affected by any of the tested salts on SBA.

All synergistic haemolysis reactions were always obtainable after an overnight incubation. Prolonged incubation increased the size of the haemolytic patterns but did not influence the appearance of $C$. sordellii synergistic haemolysis on SBA or $\mathrm{Ca}^{2+}$ unsupplemented HBA.

When used undiluted, $C$. perfringens alphaantitoxin inhibited all haemolytic activities of the four clostridial species (reactions given by some $C$. paraperfringens isolates were only partially inhibited on HBA). If diluted $1: 6$, the antitoxin completely 
inhibited all lytic activities of $C$. perfringens and synergistic lysis of $C$. bifermentans and $C$. sordellii on appropriate media. The usual and, frequently, synergistic haemolysis of $C$. paraperfringens, and the usual haemolysis of $C$. bifermentans or $C$. sordellii were only partially inhibited (Figs 1, 2, and 3). Dilution 1:10 of the antitoxin completely inhibited all lytic activities of $C$. perfringens but did not affect any of the haemolytic patterns of the other three species.

\section{Discussion}

A synergistic haemolysis test, using group B streptococci, showed an advantageous ability to identify and differentiate simply and presumptively $C$. perfringens, $C$. bifermentans, $C$. sordellii, and $C$. paraperfringens. Moreover, the test offered a double control on the same plate in that the target, or other usual haemolysis pattern, and the synergistic haemolysis were both inhibited by the same antitoxin. The use of the diluted antitoxin helped clearly to separate $C$. perfringens from $C$. bifermentans, $C$. sordellii, and $C$. paraperfringens, and CaHBA differentiated between $C$. bifermentans and $C$. sordellii. It is conceivable that different batches of $C$. perfringens type $\mathrm{A}$ antiserum should first be titrated for optimal dilution due to a possible batch-to-batch variation. Strongly haemolytic clostridial isolates should be tested with the undiluted antitoxin, and also for tryptophanase and urease production. If only $C$. perfringens is to be identified, SBA could be used alone. If all four species are to be indentified, HBA and CaHBA should be sufficient. Although the small-sized, bulletshaped, synergistic haemolysis reaction indicated the presence of $C$. paraperfringens, the statement given for the egg-yolk emulsion plate reactions ${ }^{14}$ was also applicable here. Thus, reliance could not be placed solely on the differential quantitation of the lytic zone sizes in the identification of the species. Production of phospholipase $\mathrm{C}$ varied from isolate to isolate, and in the same isolate from time to time: this caused differences in size of the reactions, which were also affected by the condition of the medium. Further, a small-sized lytic zone occurred in mixed inocula when a clostridium that was not producing phospholipase $\mathrm{C}$ predominated over $C$. perfringens. The lack of inhibition by the diluted antitoxin was much more reliable than accurate measurement of zone sizes.

The choice of the base medium and the type of blood product seems to be important. Some trials with Columbia agar base (Oxoid, London, UK) and with packed human blood bank erythrocytes gave erratic haemolytic patterns. Therefore, tryptose blood agar base and whole human blood are recommended.

Some variability in clarity of haemolysis occurred with different batches of the whole human blood bank blood. The addition of $\mathrm{Mg}^{2+}, \mathrm{Zn}^{2+}$, or $\mathrm{Ca}^{2+}$ was most beneficial to enhance uniformity of performance of HBA in that the haemolytic patterns of the tested clostridia were improved. Moreover, it indicated that $C$. sordellii phospholipase $C$ was more $\mathrm{Ca}^{2+}$ dependent than that of $C$. bifermentans, and that the enzymes of both species could not attack sphingomyelin in the outer leaflet of intact sheep erythrocytes, even when aided by $\mathrm{Mg}^{2+}$ or the CAMP-factor. In short, sphingomyelin is the major phospholipid constituent of sheep erythrocyte membrane, ${ }^{15}$ whose hydrolysis is the prerequisite for this type of haemolysis, ${ }^{16}$ and sphingomyelinase $\mathrm{C}$ activity of type $\mathrm{C}$ phosphohydrolase possessing it, depends on the presence of $\mathrm{Mg}^{2+}{ }^{1718}$ Furthermore, $C$. perfringens, whose phospholipase $\mathrm{C}$ has known sphingomyelinase activity, ${ }^{17}$ gave more intensive haemolysis on HBA and CaHBA than the former two species. Small-sized, synergistic, lytic zones of $C$. paraperfringens indicated to a small amount of phospholipase $\mathrm{C}$ produced, and its availability on both SBA and HBA suggested a similar activity to that of $C$. perfringens.

In conclusion, the synergistic haemolysis test proved simple and reliable for the presumptive identification and differentiation of $C$. perfringens, $C$. bifermentans, $C$. sordellii, and $C$. paraperfringens and may obviate the need for the Nagler test. There is an obvious economy in the use of diluted antitoxin and in the simplified differentiation of the four species. The eventual usefulness of the test in the presumptive identification of other phospholipase $\mathrm{C}$ producing clostridia has still to be determined.

I am grateful to Dr Paul Chadwick for reviewing the manuscript and for useful suggestions. My special thanks are due to Mrs Heather Dyer for excellent editorial help. Thanks are also due to $\mathrm{Dr} D E$ Mahony, Mr D Muncey, and Mr M Magus, who donated their $C$. bifermentans and $C$. sordellii strains.

\section{References}

1 Nagler FPO. Observations on a reaction between the lethal toxin of $\mathrm{Cl}$. welchii (Type A) and human serum. Br J Exp Pathol 1939;20:473-85.

${ }^{2}$ Hayward NJ. The rapid identification of $\mathrm{Cl}$. welchii by Nagler tests in plate cultures. J Pathol Bacteriol $1943 ; 55: 285-93$. 
${ }^{3}$ McClung LS, Toabe R. The egg-yolk plate reaction for the presumptive diagnosis of Clostridium sporogenes and certain species of gangrene and Botulinum groups. J Bacteriol 1947;53:139-47.

${ }^{4}$ Macfarlane MG, Knight BCJG. The biocnemistry of bacterial toxins. 1. The lecithinase activity of $\mathrm{Cl}$. welchii toxins. Biochem J 1941;35:884-902.

${ }^{5}$ Willis AT, Gowland G. Some observations on the mechanism of the Nagler reaction. J Pathol Bacteriol 1962;83:219-26.

${ }^{6}$ Nakamura S, Shimamura T, Hayase M, Nishida S. Numerical taxonomy of saccharolytic clostridia, particularly Clostridium perfringens-like strains: Descriptions of Clostridium absonum sp. n. and Clostridium paraperfringens. Int J Syst Bacteriol 1973;23:419-29.

' Smith LDS, Hobbs G. Clostridium. In: Buchanan RE, Gibbons NE, eds. Bergey's Manual of Determinative Bacteriology. 8th ed. Baltimore: Williams \& Wilkins, 1974 :553,556-7.

${ }^{8}$ Sutter VL, Vargo VL, Finegold SM. Wadsworth Anaerobic Bacteriology Manual. Los Angeles: Department of Continuing Education and Health Sciences, University Extension and School of Medicine UCLA, 1975.

${ }^{9}$ Macfarlane RG, Oakley CL, Anderson CG. Haemolysis and the production of opalescence in serum and lecitho-vitellin by the a-toxin of Clostridium welchii. J Pathol Bacteriol 1941;52:99-103.

${ }^{10}$ Gubash SM. Synergistic hemolysis phenomenon shown by an alpha-toxin-producing Clostridium perfringens and streptococcal CAMP-factor in presumptive streptococcal grouping. J Clin Microbiol 1978;8:480-8.
${ }^{11}$ Lewis GM, Macfarlane MG. The lecithinase of Clostridium bifermentans toxin. Biochem J 1953;54: 138-42.

12 Zamecnik PC, Brewster LE, Lipmann F. A manometric method for measuring the activity of the $C$. welchii lecithinase and a description of certain properties of this enzyme. J Exp Med 1947;85:381-94.

${ }^{13}$ Sato H, Murata R. Role of zinc in the production of Clostridium perfringens alpha toxin. Infect Immun $1973 ; 8: 360-9$.

${ }^{14}$ Willis AT, Hobbs G. A medium for the identification of clostridia producing opalescence in egg-yolk emulsions. J Pathol Bacteriol 1958;75:299-305.

${ }^{15}$ Nelson GJ. Lipid composition of erythrocytes in various mammalian species. Biochim Biophys Acta 1967;144: 221-32.

${ }^{16}$ Colley CM, Zwaal RFA, Roelofsen B, van Deenen LLM. Lytic and non-lytic degradation of phospholipids in mammalian erythrocytes by pure phospholipases. Biochim Biophys Acta 1973;307:74-82.

${ }^{17}$ Pastan J, Macchia V, Katzen R. A phospholipase specific for sphingomyelin from Clostridium perfringens. J Biol Chem 1968;24(3):3750-5.

${ }^{18}$ Low DKR, Freer JH, Arbuthnott JP. Consequences of sphingomyelin degradation in erythrocyte ghost membranes by staphylococcal $\beta$ toxin (sphingomyelinase C). Toxicon 1974;12:279-85.

Requests for reprints to: $\mathrm{Dr}$ SM Gubash, Provincial Laboratory of Public Health, Southern Branch, PO Box 2490, Calgary, Alberta, Canada T2P 2M7. 